\title{
PIGMENTOS FOLIARES NA CULTURA DA ERVILHA: RELAÇÃO ENTRE ÍNDICE SPAD E CONTEÚDO DE CLOROFILA
}

\author{
LEAF PIGMENTS IN PEA CROP: RELATIONSHIP BETWEEN SPAD INDEX AND \\ CHLOROPHYLL CONTENT \&
}
PIGMENTOS FOLIARES EN CULTIVOS DE GUISANTES: RELACIÓN ENTRE EL ÍNDICE SPAD Y EL CONTENIDO DE CLOROFILA \&

Recebido em: 23/07/2021 - Aprovado em: 20/10/2021 - Publicado em: 07/11/2021

doi) http://dx.doi.org/10.18011/bioeng2021v15n3p391-400

Gustavo Soares Wenneck' (gustavowenneck@gmail.com)

Vinicius Villa e Vila1 (vinivilla95@hotmail.com)

Reni Saath1 (rsaath@uem.br)

Roberto Rezende ${ }^{1}$ (rrezende@uem.br)

Gustavo Lopes Pereira' ${ }^{1}$ (gustavolopespereira@hotmail.com)

Bruna Mayara Conti Mondanez ${ }^{1}$ (mayara_conti@hotmail.com)

\footnotetext{
${ }^{1}$ Universidade Estadual de Maringá. Maringá, PARANÁ, Brasil.
}

\section{RESUMO}

Os teores de pigmentos foliares estão diretamente relacionados com as condições de desenvolvimento da planta, sendo a determinação realizada por métodos diretos e indiretos. $O$ trabalho teve como objetivo relacionar os valores do índice SPAD com o conteúdo de pigmentos foliares na cultura da ervilha. A determinação foi realizada em folhas de plantas em estágio reprodutivo, apresentando coloração variando de amarelada para verde escuro. A determinação indireta foi realizada com o equipamento SPAD 502 e a determinação direta por espectrometria utilizando acetona pura como extrator. Foi realizada análise descritiva, correlação e de regressão nos dados. O índice SPAD obtido variou entre 8,2 e 42,2. O teor absoluto de clorofila é elevado em valores de SPAD superiores, ajustado por modelos quadráticos. Foi obtido elevada correlação entre índice SPAD e pigmentos foliares (clorofilas $a, b$ e totais) em folhas de ervilha.

Palavras-chave: Análise de métodos. Pigmentos foliares. Pisum sativum. 


\section{INTRODUÇÃO}

Os pigmentos foliares, principalmente clorofila e carotenoides, são reflexo da atividade fotossintética e das condições de desenvolvimento da planta, envolvendo nutrição, disponibilidade hídrica e sombreamento (JIANG et al., 2017; SHAH et al., 2017; SILVA, 2020). A quantificação do conteúdo de clorofila pode envolver métodos diretos, com a extração dos pigmentos, e métodos indiretos, com a determinação pelo índice SPAD (SHIBAEVA et al., 2020; WENNECK et al., 2021).

No cultivo da ervilha, o desenvolvimento morfológico e potencial produtivo está relacionado a eficiência fotossintética, com relação direta do índice SPAD (FERHI et al., 2017). Entretanto, o emprego de metodologias para avaliação da relação entre índice SPAD e conteúdo absoluto de clorofila é necessário para obtenção de modelos matemáticos de predição com estimativas adequadas (JIANG et al., 2017; MAIA JÚNIOR et al., 2017; NOGUEIRA et al., 2018).

A caracterização dos pigmentos foliares em função do índice SPAD é empregada em diversas espécies, a fim de possibilitar a adoção do recurso para eficiência do sistema produtivo (DANIEL et al., 2016; SHAH et al., 2017; DIAS et al., 2020; VIDIGAL et al., 2021). Dessa forma, o trabalho teve como objetivo relacionar os valores do índice SPAD com o conteúdo de pigmentos foliares na ervilha.

\section{MATERIAIS E MÉTODOS}

O estudo foi desenvolvido no Centro Técnico de Irrigação (CTI) da Universidade Estadual de Maringá (UEM). O experimento foi conduzido em delineamento inteiramente casualizado, com 40 amostras de folhas sendo determinado o índice SPAD e o conteúdo de pigmentos foliares.

Foram utilizadas folhas do terço superior de plantas de ervilha, durante o florescimento, cultivada em NITOSSOLO VERMELHO distroférrico (SANTOS et al., 2018). A semeadura da cultura ocorreu entre os meses de maio a junho de 2021. As determinações a campo e coletas de amostras foram realizadas no dia 17 de agosto de 2021 , entre as 9 e 10 horas com temperatura média de $20^{\circ} \mathrm{C}$ e umidade relativa de $82 \%$. As determinações ocorreram de folhas selecionadas de forma aleatória, com intensidade de cor variando entre amarelada a verde-escuro. 
A determinação do índice SPAD foi realizada com o equipamento SPAD-502 $\left(\right.$ Minota $\left.{ }^{\circledR}\right)$ com três leituras simultâneas e posterior coleta da folha para determinação dos pigmentos. Os pigmentos foliares, clorofila e carotenoides, foram determinados por espectrometria utilizando acetona pura como extrator. As amostras de tecido foliar (150 mg) foram mantidas submersas em acetona $(2 \mathrm{~mL})$ no escuro e em baixa temperatura até despigmentação do tecido. Foi realizada a leitura do extrato em cubetas de quartzo em espectrofotômetro nos comprimentos de onda de 661,4; 644,8 e 470 nm, sendo determinado os teores de clorofila $a$, clorofila $b$, clorofila total e carotenoides conforme Lichtenthaler (1987).

Foi calculada a média aritmética das leituras com o SPAD-502 obtendo-se um valor para cada amostra. Foi realizada análise descritiva dos dados, correlação linear entre as variáveis e análise de regressão entre pigmentos foliares e índice SPAD. Para análise dos dados foram utilizados os softwares SISVAR (Ferreira, 2019) e Microsoft Excell@.

\section{RESULTADOS E DISCUSSÃO}

Conforme análise descritiva (Tabela 1) foram obtidos valores de índice SPAD entre 8,2 e 42,2, sendo obtido principalmente valores elevados considerando a assimetria negativa dos dados.

Tabela 1 - Análise descritiva dos valores de índice SPAD

\begin{tabular}{c|c|c|c}
\hline Parâmetro & Valor & Parâmetro & Valor \\
\hline Média & 28,82 & Curtose & $-0,879$ \\
\hline Erro padrão & 1,73 & Assimetria & $-0,599$ \\
\hline Mediana & 32,1 & Intervalo & 34 \\
\hline Modo & 37 & Mínimo & 8,2 \\
\hline Desvio padrão & 9,964 & Máximo & 42,2 \\
\hline
\end{tabular}

Fonte: Os autores, 2021.

A partir da determinação dos parâmetros por métodos destrutivo e não destrutivo foram obtidos modelos matemáticos para predição com base no índice SPAD no conteúdo de clorofila a (Figura 1A), clorofila b (Figura 1B), clorofila total (Figura 1C) e carotenóides (Figura 1D). O índice SPAD pode ser adotado para determinação de conteúdo de clorofila mesmo com presença de clorose nas folhas avaliadas (SHIBAEVA et al., 2020). 
Figura 1 - Conteúdo de pigmentos foliares em função do indice SPAD em folhas de ervilha. A)

Clorofila a; B) Clorofila b; C) Clorofila total; D) Carotenoides
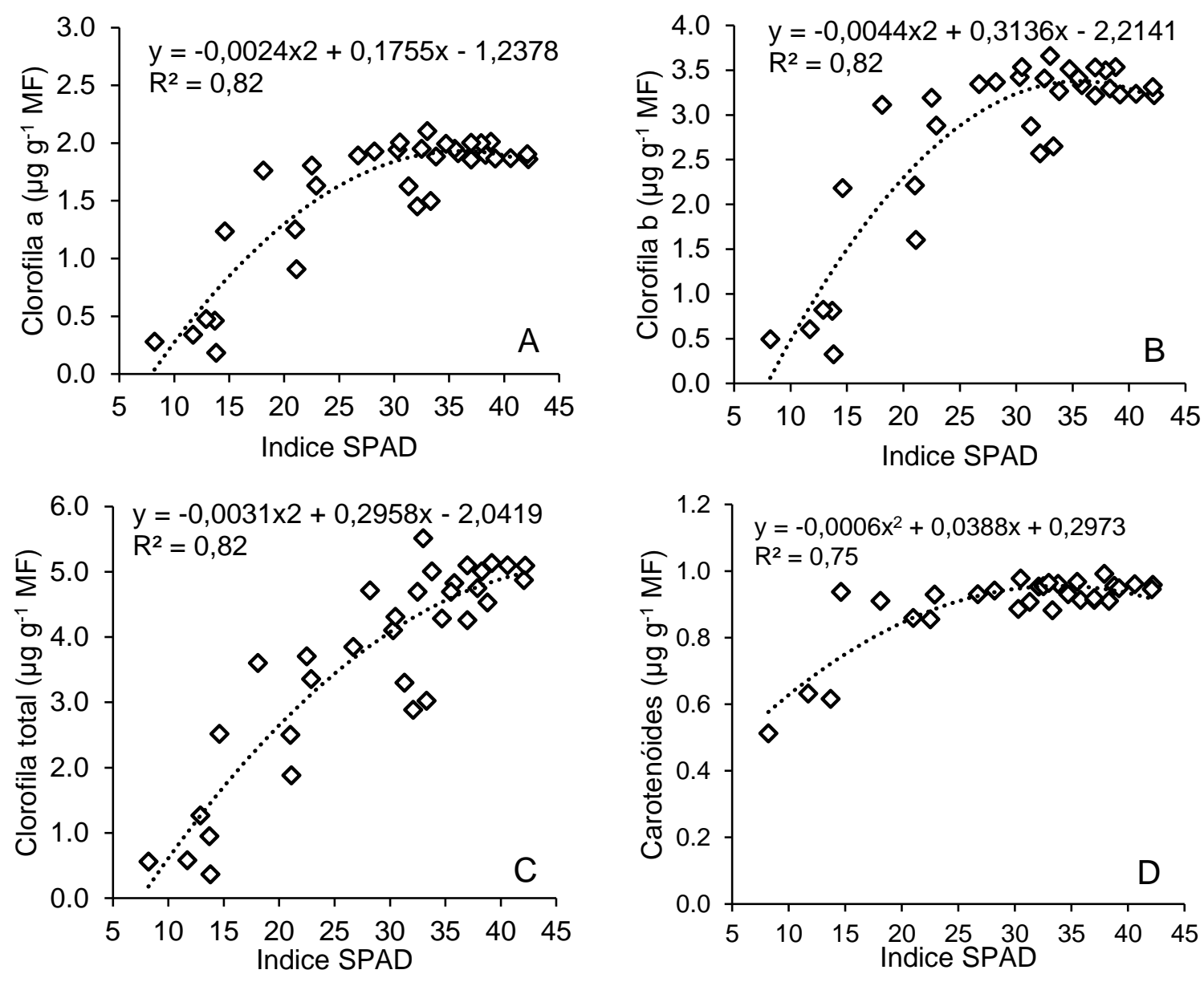

Fonte: Os autores, 2021.

O coeficiente de determinação das equações (Figura 1) variou entre 0,75 e 0,82 sendo próximo ao obtido por Daniel et al. (2016) em Salix viminalis ao analisar a relação do índice SPAD e teores absolutos em função da massa fresca foliar, com coeficiente entre 0,79 e 0,81. Embora no estudo foram realizadas determinações apenas durante estádio reprodutivo da ervilha, variações na relação de pigmentos foliares podem ser obtidas nos diferentes estádios de desenvolvimento da planta, sendo fator importante no ajuste de modelos matemáticos para predição da relação (JIANG et al., 2017).

Os valores de índice SPAD refletem características fisiológicas e nutricionais das plantas, sendo responsivos a condições de estresse hídrico (SHAH et al., 2017). Na cultura da ervilha, o índice SPAD tem relação direta com a atividade fotossintética e crescimento da planta (FERHI et al., 2017). 
Foi observado variação na relação carotenoide/clorofila total em função do índice SPAD obtido, sendo que a relação reduz com o acréscimo do índice SPAD (Figura 2). A relação do conteúdo de carotenoides e clorofila está relacionado a fisiologia vegetal, em que incremento no teor de carotenoides são resposta de fotoproteção da planta em condições desfavoráveis (SILVA, 2020).

Figura 2 - Relação entre carotenoides e clorofila total em função do índice SPAD em folhas de ervilha.

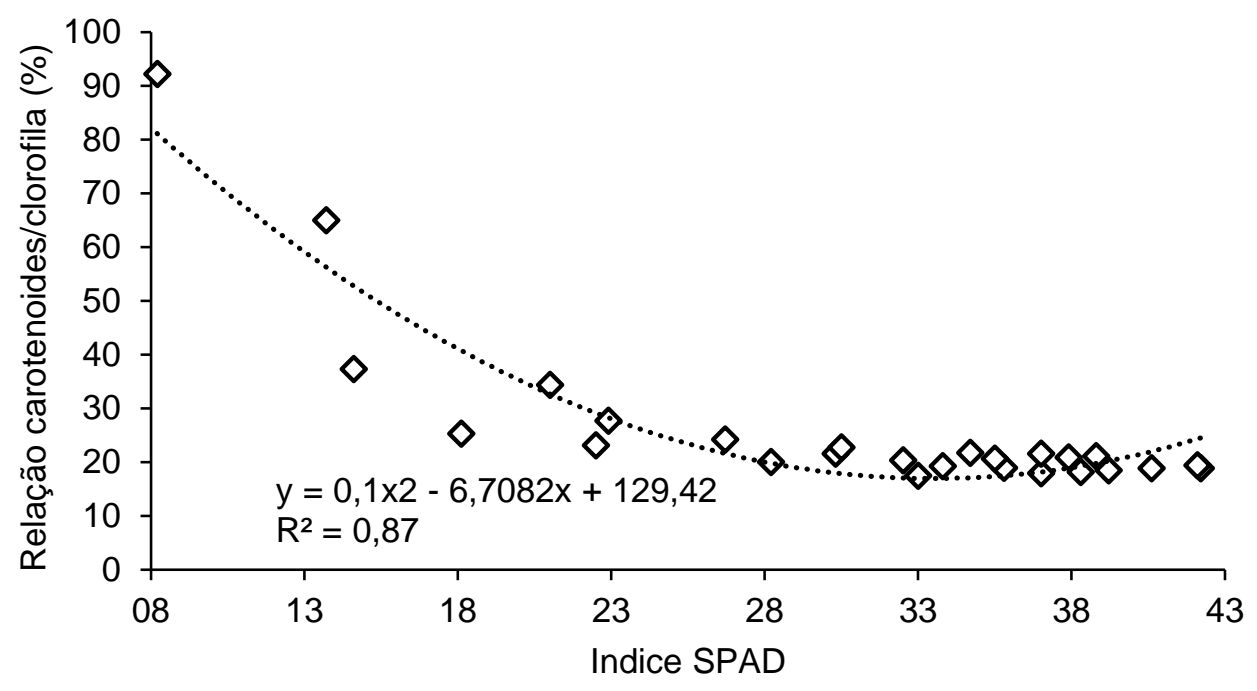

Fonte: Os autores, 2021.

Ao realizar a amostragem das folhas de forma aleatória na área de cultivo, condições desfavoráveis antes não identificadas e isoladas foram detectadas nos resultados obtidos. A relação carotenoide/clorofila inferior a $25 \%$ foi obtida com índice SPAD superior a 27 (Figura 2).

Embora estimada a relação com conteúdo de carotenoides, a utilização do SPAD 502 é realizada principalmente para determinação de clorofila ( $a, b$ e total) com confiabilidade elevada quando considerado a relação entre área foliar e massa do tecido analisado (DANIEL et al., 2016).

Os valores de correlação entre índice SPAD e clorofilas foi superior a 0,8 e para carotenoides de 0,74 (Tabela 2). O emprego da análise de correlação foi realizado considerando os dados globais, porém na presença de condições de desenvolvimento diferentes para uma mesma espécie, a análise pode ser adotada para avaliar o impacto da condição sobre as variáveis. Wenneck et al. (2021) obtiveram variações na interação do 
índice SPAD com os pigmentos, com variação da clorofila a e b variável com a disponibilidade hídrica e adubação.

Tabela 2 - Correlação linear para pigmentos foliares e índice SPAD

\begin{tabular}{c|c|c|c|c|c}
\hline & Clorofila a & Clorofila b & Clorofila total & Carotenoides & SPAD $^{(1)}$ \\
\hline Clorofila a & 1,00 & - & - & - & - \\
\hline Clorofila b & 0,98 & 1,00 & - & - & - \\
\hline Clorofila total & 0,96 & 0,95 & 1,00 & - & - \\
\hline Carotenoides & 0,91 & 0,91 & 0,85 & 1,00 & \\
\hline SPAD $^{(1)}$ & 0,83 & 0,83 & 0,89 & 0,74 & 1,00
\end{tabular}

(1) Índice SPAD.

Fonte: Os autores, 2021.

Considerando a elevada correção nos valores obtidos entre método direto e indireto, os valores podem ser utilizados para o manejo nutricional da cultura (PÔRTO et al., 2011) e como critério para seleção em programas de melhoramento genético (SILVA et al., 2014). A determinação do conteúdo de pigmentos foliares pode também ser adotada como parâmetro pós-colheita de hortaliças folhosas (DIAS et al., 2020).

A quantificação de pigmentos foliares com SPAD permite definir estratégias para manejo da cultura (PÔRTO et al., 2011; NOGUERIA et al., 2018;). Entretanto, as relações são influenciadas pela espécie, condições edafoclimáticas e tratos culturais (MAIA JUNIOR et al., 2017; VIDIGAL et al., 2021). O ajuste de modelos matemáticos entre índice SPAD e teores de pigmentos foliares permite a predição de níveis absolutos em ervilha, auxiliando a obtenção de dados com rapidez e precisão.

\section{CONCLUSÕES}

O índice SPAD apresentou correlação com os teores de clorofila a, clorofila b e clorofila total em folhas de ervilha.

A relação carotenoide/clorofila é inferior com valores elevados de índice SPAD. 


\section{REFERÊNCIAS}

DANIEL, E. S.; AMARANTE, C. V. T.; MARTIN, M. S.; MIQUELLUTI, D. J.; CAMPOS, M. L. Relação entre o teor absoluto e relativo de clorofila em folhas de vimeiro. Ciência Florestal, v. 26, n. 1, p. 307-312, 2016. DOI: 10.5902/1980509821122

DIAS, L. A.; SILVEIRA, P. H. A. M.; OMURA, S. S.; GARCIA, B.; OLIVEIRA, R. E. V.; PEREIRA, M. S. C. Determinação de Teores de Clorofilas e Carotenoides em Alface, Rúcula e Cebolinha. Brazilian Journal of Animal and Environmental Research, v. 3, n. 4, p. 3100-3107, 2020. DOI: 10.34188/bjaerv3n4-030

FERREIRA, D. F. SISVAR: a computer analysis system to fixed effects split plot type designs. Revista Brasileira de Biometria, v. 37, n.4, p. 529-535, 2019.

FERHI, J.; GHARSALLI, M.; ABDELLY, C.; KROUMA, A. Potential of the physiological response of pea plants (Pisum sativum L.) to iron deficiency (direct or lime- induced). Bioscience Journal, v.33, n.5, p.1208-1218, 2017. DOI: 10.14393/BJ-v33n5a2017-36988

JIANG, C.; JOHKAN, M.; HOHJO, M.; TSUKAGOSHI, S.; MARUO, T. A correlation analysis on chlorophyll content and SPAD value in tomato leaves. HortResearch, n.71, p.37-42, 2017. DOI: 10.20776/S18808824-71-P37

LICHTENTHALER, H. K. Chlorophylls and carotenoids: pigments of photosynthetic biomembranes. Methods in Enzymology, New York, v.148, p.350-382, 1987.

MAIA JÚNIOR, S. O.; ANDRADE, J. R.; FERREIRA, R. S.; ARAÚJO, D. L.; GUERRA, H. O. C.; SILVA, F. G. Teores de pigmentos, fluorescência da clorofila a e índice SPAD em cultivares de girassol sob regimes hídricos. Revista Agrarian, v.10, n.36, p.105-112, 2017. DOI: 10.30612 /agrarian.v10i36.3604

NOGUEIRA, B. B.; IGLESIAS, L.; MESQUITA, J. V.; NAKATANI, M. C.; PUTTI, F. F. Índice SPAD em plantas de tomateiro cultivado em fibra de coco e submetido a pulsos de fertirrigação. Brazilian Journal of Biosystems Engineering, v. 12, n.1, p.1-6, 2018. DOI: 10.18011/bioeng2018v12n1p1-6

PÔRTO, M. L; PUIATTI, M.; FONTES, P. C. R.; CECON, P. R.; ALVES, J. C.; ARRUDA, J. A. Índice SPAD para o diagnóstico do estado de nitrogênio na cultura da abobrinha. Horticultura Brasileira, v.29, n.3, p. 311-315, 2011. DOI: 10.1590/S010205362011000300009

SANTOS, H. G.; JACOMINE, P. K. T.; ANJOS, L. H. C.; OLIVEIRA, V. Á.; LUMBRERAS, J. F.; COELHO, M. R.; ALMEIDA, J. A.; ARAÚJO FILHO, J. C.; OLIVEIRA, J. B.; CUNHA, T. J. F. Sistema Brasileiro de Classificação de Solos. 5. ed. Brasília, DF: EMBRAPA, 2018.

SHAH, S. H.; HOUBORG, R.; MCCABE, M. F. Response of Chlorophyll, Carotenoid and SPAD-502 Measurement to Salinity and Nutrient Stress in Wheat (Triticum aestivum L.). Agronomy, v.7, n.3, e.61, 2017. DOI: 10.3390/agronomy7030061 
SHIBAEVA, T. G.; MAMAEV, A. V.; SHERUDILO, E. G. Evaluation of a SPAD-502 Plus Chlorophyll Meter to Estimate Chlorophyll Content in Leaves with Interveinal Chlorosis. Russian Journal of Plant Physiology, v.67, p.690-696, 2020. DOI:

$10.1134 /$ S1021443720040160

SILVA, L. R. Alterações morfofisiológicas em feijoa (Acca sellowiana) sob diferentes níveis de sombreamento. 2020. 79f. Dissertação (Mestrado) - Universidade Tecnológica Federal do Paraná -UTFPR, Pato Branco.

SILVA, M. A.; SANTOS, C. M.; VITORINO, H. S.; RHEIN, A. F. L. Pigmentos fotossintéticos e índice SPAD como descritores de intensidade do estresse por deficiência hídrica em cana-de-açúcar. Bioscience Journal, v. 30, n. 1, p. 173-181, 2014.

VIDIGAL, S. M.; PUIATTI, M.; LOPES, I. P. C.; SEDIYAMA, M. A. N. Nitrogen content, SPAD index and production of single head broccoli. Horticultura Brasileira, v.39, n.1, p.52-57, 2021. DOI:10.1590/s0102-0536-20210108

WENNECK, G. S.; SAATH, R.; REZENDE, R.; SILVA, L. H. M. SPAD index and leaf pigments in cauliflower in different water conditions and silicon fertilization. Revista Engenharia na Agricultura, v.29, p.204-210, 2021. DOI:10.13083/reveng.v29i1.12460 


\begin{abstract}
The contents of leaf pigments are directly related to the conditions of plant development, and the determination is carried out by direct and indirect methods. The aim of this work was to relate the values of the SPAD index with the content of leaf pigments in the pea crop. The determination was carried out on plant's leaves in the reproductive stage, varying the color from yellowish to dark green. The indirect determination was performed with the SPAD 502 equipment and the direct determination by spectrometry using pure acetone as an extractor. Descriptive, correlation and regression analysis were performed on the data. The SPAD index obtained ranged between 8.2 and 42.2. The absolute chlorophyll content is high at higher SPAD values, adjusted by quadratic models. High correlation was obtained between SPAD index and leaf pigments ( $a, b$ and total chlorophylls) in pea leaves.
\end{abstract}

Keywords: Methods analysis. Leaf pigments. Pisum sativum.

\title{
RESUMEN
}

El contenido de los pigmentos foliares está directamente relacionado con las condiciones de desarrollo de la planta, la determinación se realiza por métodos directos y indirectos. El objetivo de este trabajo fue relacionar los valores del índice SPAD con el contenido de pigmentos foliares en cultivos de guisantes. La determinación se realizó en hojas de plantas en etapa reproductiva, con color que variaba de amarillento a verde oscuro. La determinación indirecta se realizó con el equipo SPAD 502 y la determinación directa por espectrometría utilizando acetona pura como extractor. Se realizó análisis descriptivo, de correlación y regresión de los datos. El índice SPAD obtenido osciló entre 8,2 y 42,2. El contenido absoluto de clorofila es alto a valores más altos de SPAD, ajustado por modelos cuadráticos. Se obtuvo una alta correlación entre el índice SPAD y los pigmentos foliares (clorofilas a, b y totales) en hojas de guisante.

Palabras-clave: Análises de métodos. Pigmentos foliares. Pisum sativum. 


\section{LICENÇA DE USO}

Este é um artigo publicado em acesso aberto (Open Access) sob a licença Creative Commons Atribuição 4.0 Internacional (CC BY 4.0), que permite uso, distribuição e reprodução em qualquer meio, desde que o trabalho original seja corretamente citado. Mais informações em: http://creativecommons.org/licenses/by/4.0

\section{CONFLITO DE INTERESSES}

Os autores declaram que não há conflito de interesses neste trabalho.

\section{CONTRIBUIÇÕES AUTORAIS}

Autor 1: planejamento experimental, análise de dados e redação do manuscrito.

Autor 2: planejamento experimental, coleta de dados, análise de dados e revisão do manuscrito.

Autor 3: supervisão e orientação.

Autor 4: supervisão e orientação.

Autor 5: correção e revisão do manuscrito.

Autor 6: coleta de dados.

\section{AGRADECIMENTO}

A Coordenação de Aperfeiçoamento de Pessoal de Nível Superior (CAPES), ao Conselho Nacional de Desenvolvimento Científico e Tecnológico (CNPq) e a Universidade Estadual de Maringá (UEM).

\section{COMO REFERENCIAR}

WENNECK, Gustavo Soares et al. Pigmentos foliares na cultura da ervilha: relação entre índice spad e conteúdo de clorofila. Revista Brasileira de Engenharia de Biossistemas (Tupã), v. 15, n. 3, p. 391-400, 2021. DOI:

http://dx.doi.org/10.18011/bioeng2021v15n3p391-400.

\section{RESPONSABILIBADE EDITORIAL}

Prof. Dr. Fernando Ferrari Putti ${ }^{1}$, Prof. Dr. Paulo Sérgio Barbosa dos Santos ${ }^{1}$, Prof. Dr. Eduardo Festozo Vicente ${ }^{1}$ e Prof. Dr. Diogo de Lucca Sartori ${ }^{1}$

${ }^{1}$ Universidade Estadual Paulista "Júlio de Mesquita Filho", FCE - Faculdade de Ciências e Engenharia, Tupã, SP, Brasil. 